\title{
Nonlinear robust control for single-machine infinite-bus power systems with input saturation
}

\author{
Y. WAN* \\ College of Automation Engineering, Nanjing University of Aeronautics and Astronautics, Nanjing 211106, China
}

\begin{abstract}
In this paper, a new control scheme is proposed to achieve stability for a single-machine infinite-bus power system. A power system model simultaneously considering input saturation and time-varying uncertainties is presented. A sufficient condition for the system convergence is given and based on this result, a switching excitation control law with auxiliary system is designed. The stability analysis and simulation results all show that the developed controller is effective.
\end{abstract}

Key words: nonlinear robust control, input saturation, generator excitation, single-machine infinite-bus power systems (SMIB).

\section{Introduction}

Energy issue is one of the most pressing challenges. The main contents of this problem include generation, transmission and distribution of energy. Power system is regarded as one of the most complex systems in the world [1] and in order to obtain satisfactory and optimal operating state, many researchers have developed various solutions from their different professional perspectives [2-5]. This paper studies power system transient stability by using model-based control systems technology [6].

For many years, linear control has been used as a traditional soft computing method to design excitation controllers, e.g. automatic voltage regulator (AVR) and power system stabilizer (PSS). Although these controllers have been simulatively validated, the robustness can not be ensured because the controllers have been designed on the basis of linear power system model which is often not applicable in entire operation region. Thus it is necessary to preserve nonlinear characteristics of power systems and study nonlinear control method. However, there are strongly nonlinear couplings between model parameters and state variables, and the known values of the parameters are often not coincident with the actual situation. Thus, model errors are always subsistent and are necessary to be considered in control system synthesis from the viewpoints of nonlinear robust control $[7,8]$. Furthermore, input constraints, such as excitation voltage saturation, are unavoidable in practical engineering. If this phenomenon is not considered in controller design, the prospective stability and performances of closedloop systems may not be ensured because the system dynamic behavior is uncontrolled when the desired control input goes beyond the limit.

Due to the complex nature of nonlinear systems, in the existing literatures, most of results on input saturation/constraints are based on linear systems. Only few researchers pay atten-

*e-mail: wanyong@nuaa.edu.cn tion to the control problem of nonlinear systems with input saturation. In [9], an auxiliary system is employed to address input constraints, but only boundedness of the "last modified virtual error" is obtained. If the desired value of control input greatly exceeds the constraints, then the auxiliary system will be unstable and thus the convergence of the "last virtual error" to the origin cannot be ensured. In [10], the tanh function and the mean-value theorem are used to deal with the saturation function, and then the redial basis function i.e. neural network is employed to approximate the unknown control gain. However, since the tanh function is also a kind of bounded function, when there are large disturbances and the desired value of control input goes beyond the range of tanh, the efficacy of the designed control law may be limited. In [11], dynamic surface control is used to equivalently transform a class of nonlinear system into a linear system, and then the region of attraction which is enlarged to allow some degree of input saturation is estimated via a linear matrix inequality method [12]. But this approach does not give a solution for a case when the initial condition is out of this region of attraction.

This article proposes a new nonlinear robust control framework to tackle the stabilization problem for a class of nonlinear system with time-varying uncertainties and input saturation. Compared with the prior works, the proposed method ensures not only the boundedness of state variables but the global stability of the closed-loop system. Furthermore, the approach proposed processes the saturation phenomenon directly and does not require approximating the saturation function by a smooth function, which is often not invertible outside its range.

The paper is organized as follows: the preliminaries and a SMIB power system model are described in Section 2. In Section 3, a sufficient condition for the convergence of states to the origin under saturation is shown in a Theorem, and then on the basis of this result, a switching excitation control law with auxiliary system (SAEC) is designed, as well as stability analysis for the closed-loop system with the designed controller. The simulation results are presented in Section 4. Conclusions are drawn in Section 5. 


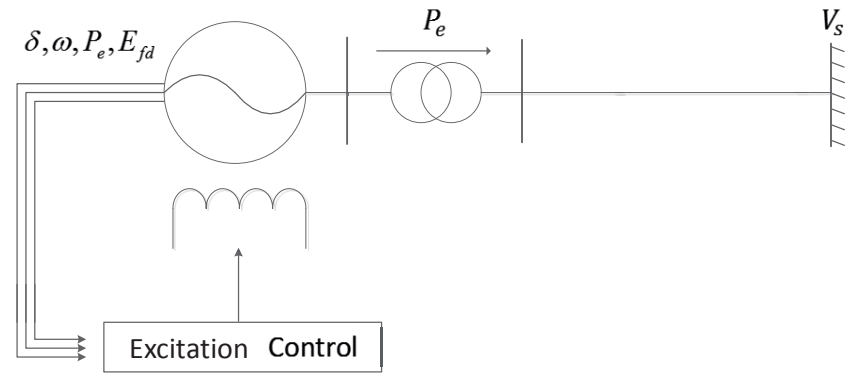

Fig. 1. Single-machine infinite-bus power systems

\section{Problem statement and system model}

Consider the SMIB system with its components as shown in Fig. 1. Compared with structure-preserving multi-machine model $[13,14]$, the SMIB model does not directly reflect the influences of power network components and loads, instead of aggregated node containing the information of these elements. Furthermore, the reduced-order model also neglects the flux linkages dynamics by using the singular perturbation technique. Fortunately, even so, both the theoretical studies and the experimental results have validated the SMIB model are well-suited for stability analysis and feedback control design for industrial power generators $[15,16]$. Based on the above discussions, from the perspective of robust control, in order to enhance the applicability of excitation control system, time-varying uncertainties indicating the model errors should be considered. Moreover, in practice, actuator saturation is ubiquitous and there exist input constraint. Simultaneously ensuring the expected performances and the control laws remaining within bounds is difficult, and in this case, it is a critical and challenging problem for state-feedback control of power systems with input saturation. As such, a nonlinear robust excitation control scheme considering excitation input constraint is proposed in this paper.

Thus, on the basis of the classical SMIB model $[17,18]$ and the above statements, the system dynamic model considered in this paper is proposed as follows:

$$
\begin{aligned}
& \dot{\delta}=\omega, \\
& \dot{\omega}=-(D / H) \omega+\left(\omega_{0} / H\right)\left(P_{m}-P_{e}\right)+d_{1}(t), \\
& \dot{P}_{e}=P_{1}(\delta, \omega) P_{e}+P_{2}(\delta)+P_{3}(\delta) \operatorname{sat}\left(E_{f d}\right)+d_{2}(t), \\
& \operatorname{sat}\left(E_{f d}\right)=\gamma E_{f d}, \\
& \qquad \gamma= \begin{cases}U_{B} /\left|E_{f d}\right|, & \left|E_{f d}\right| \geq U_{B}, \\
1, & \left|E_{f d}\right|<U_{B},\end{cases}
\end{aligned}
$$

where $\delta$ is the power angle in $\mathrm{rad}, \omega$ is the relative speed in $\mathrm{rad} / \mathrm{s}, P_{e}$ is the active power in p.u., $E_{f d}$ is the field excitation voltage in p.u., $U_{B}>0$ is a bound of the field excitation voltage, $d_{i}(t)$ are bounded time-varying uncertainties, that is, there exist unknown constants $d_{i}^{*}>0$ such that $\left|d_{i}(t)\right| \leq d_{i}^{*}$, $i=1,2 ; P_{m}$ is the mechanical power in p.u., $D$ is the damping coefficient in p.u., $H$ is the inertia constant in s, $\omega_{0}$ is the synchronous speed in $\mathrm{rad} / \mathrm{s}, T_{d 0}$ and $T_{d 0}^{\prime}$ are the time constants of the excitation winding in $\mathrm{s}, x_{d}$ is the $d$-axis synchronous reactance in p.u., $x_{d}^{\prime}$ is the $d$-axis transient reactance of the generator in p.u., $x_{T}$ and $x_{L}$ are the transformer and the line reactances in p.u., $V_{s}$ is the infinite bus voltage in p.u., $x_{d s}^{\prime}=x_{d}^{\prime}+x_{T}+x_{L}$, $c_{x d}=\left(x_{d}-x_{d}^{\prime}\right) V_{s}^{2} /\left(2 T_{d 0} x_{d s}^{\prime 2}\right), c_{x d}^{\prime}=V_{s} /\left(T_{d 0} x_{d s}^{\prime}\right), P_{1}(\delta, \omega)=$ $-1 / T_{d 0}^{\prime}+\omega \cot \delta, P_{2}(\delta)=c_{x d} \sin 2 \delta, P_{3}(\delta)=c_{x d}^{\prime} \sin \delta$.

\section{Preliminaries and controller design}

Before presenting the designed controller, some preliminaries and the preprocessing of control are given below.

3.1. Preliminaries. Generally, power systems are under normal operating conditions rather than leading power factor operations which are the rare cases $[3,19]$, thus there exists a small constant $\delta_{m}$ such that $\pi / 2>\delta>\delta_{m}>0$.

Schur complements [12]. The inequality $\left[\begin{array}{cc}\mathbf{Q}(\mathbf{x}) & \mathbf{S}(\mathbf{x}) \\ \mathbf{S}(\mathbf{x})^{\mathrm{T}} & \mathbf{R}(\mathbf{x})\end{array}\right]>0$ is equivalent to $\mathbf{Q}(\mathbf{x})-\mathbf{S}(\mathbf{x}) \mathbf{R}(\mathbf{x})^{-1} \mathbf{S}(\mathbf{x})^{\mathrm{T}}>0$, where $\mathbf{x} \in \mathbb{R}^{n}$, $\mathbf{Q}(\mathbf{x})=\mathbf{Q}(\mathbf{x})^{\mathrm{T}} \in \mathbb{R}^{m \times m}, \quad 0<\mathbf{R}(\mathbf{x})=\mathbf{R}(\mathbf{x})^{\mathrm{T}} \in \mathbb{R}^{s \times s}$, and $\mathbf{S}(\mathbf{x}) \in \mathbb{R}^{m \times s}$ depend affinely on $\mathbf{x}$.

Definition 1. The set $C(\mathbf{P}, \rho)=\left\{\mathbf{x} \mid \mathbf{x}^{\mathrm{T}} \mathbf{P} \mathbf{x} \leq \rho\right\}$ is said to be invariant ellipsoid when the following condition holds: For a trajectory $\mathbf{x}(t)$, if $\mathbf{x}(0) \in C(\mathbf{P}, \rho)$ then $\mathbf{x}(t) \in C(\mathbf{P}, \rho)$, where $0<\mathbf{P}=\mathbf{P}^{\mathrm{T}} \in \mathbb{R}^{n \times n}$ and $\rho>0$.

Definition 2. The set $C$ is said to be a region of attraction when the following condition holds: For a trajectory $\mathbf{x}(t)$, if $\mathbf{x}(0) \in C$ then $\mathbf{x}(t) \rightarrow 0$ as $t \rightarrow \infty$.

Lemma 1. Consider $\mathbf{u}=\mathbf{K} \mathbf{x} \in \mathbb{R}^{n_{u}}, \mathbf{K} \in \mathbb{R}^{n_{u} \times n}$, and $C(\mathbf{P}, \rho)$ is an invariant ellipsoid. Then $\|\mathbf{u}\| \leq u_{c}$ is equivalent to the inequalities $\left[\begin{array}{cc}\rho & \mathbf{x}(0)^{\mathrm{T}} \\ \mathbf{x}(0) & \mathbf{P}^{-1}\end{array}\right] \geq 0$ and $\left[\begin{array}{cc}\mathbf{P} & \mathbf{K}^{\mathrm{T}} \\ \mathbf{K} & \left(u_{c}^{2} / \rho\right) \mathbf{I}\end{array}\right] \geq 0$ hold.

Proof. Based on the Schur complements, $\left[\begin{array}{cc}\rho & \mathbf{x}(0)^{\mathrm{T}} \\ \mathbf{x}(0) & \mathbf{P}^{-1}\end{array}\right] \geq 0$ and $\left[\begin{array}{lc}\mathbf{P} & \mathbf{K}^{\mathrm{T}} \\ \mathbf{K} & \left(u_{c}^{2} / \rho\right) \mathbf{I}\end{array}\right] \geq 0$ can be equivalently transformed into $\mathbf{x}(0)^{\mathrm{T}} \mathbf{P} \mathbf{x}(0) \leq \rho$ and $\mathbf{P}^{-1 / 2} \mathbf{K}^{\mathrm{T}} \mathbf{K} \mathbf{P}^{-1 / 2} \leq\left(u_{c}^{2} / \rho\right) \mathbf{I}$. Thus, $\mathbf{x}(0) \in C(\mathbf{P}, \rho)$ which is an invariant ellipsoid, one can get $\mathbf{x}^{\mathrm{T}} \mathbf{P} \mathbf{x} \leq \rho$. Therefore, $\|\mathbf{u}\|^{2}=\mathbf{u}^{\mathrm{T}} \mathbf{u}=\mathbf{x}^{\mathrm{T}} \mathbf{P}^{1 / 2}\left(\mathbf{P}^{-1 / 2} \mathbf{K}^{\mathrm{T}} \mathbf{K} \mathbf{P}^{-1 / 2}\right) \mathbf{P}^{1 / 2} \mathbf{x} \leq\left(u_{c}^{2} / \rho\right) \mathbf{x}^{\mathrm{T}} \mathbf{P} \mathbf{x} \leq u_{c}^{2}$. This concludes the proof.

Definition 3 [11]. For $\varsigma \in(0,1]$, the constraint $\|\mathbf{u}\| \leq u_{c} / \varsigma$ is an extended version of $\|\mathbf{u}\| \leq u_{c}$, in this case, some degree of saturation is allowed, namely " $\zeta$-saturation".

Lemma 2. " $\zeta$-saturation" is equivalent to the inequalities $\left[\begin{array}{cc}\rho & \mathbf{x}(0)^{\mathrm{T}} \\ \mathbf{x}(0) & \mathbf{P}^{-1}\end{array}\right] \geq 0$ and $\left[\begin{array}{cc}\mathbf{P} & { }_{\zeta \mathbf{K}^{\mathrm{T}}} \\ \boldsymbol{K}_{\left(u_{c}^{2} / \rho\right) \mathbf{I}} /{ }^{-}\end{array}\right] \geq 0$ hold.

Proof. Based on Lemma 1 and the Schur complements, one can directly get the above result. 
Lemma 3. [20] For any $\varepsilon>0$ and $e \in \mathbb{R}$, the following inequality always holds

$$
0 \leq|e|-e \tanh (e / \varepsilon) \leq 0.2785 \varepsilon .
$$

3.2. SAEC control scheme. Let $\left(\delta^{*}, 0, P_{m}\right)$ and $z_{i}$ be the desired operating point of $\left(\delta, \omega, P_{e}\right)$ and the error variables respectively, $\overline{\mathbf{z}}_{\mathbf{i}}=\left[z_{1}, \ldots, z_{i}\right]^{\mathrm{T}}, i=1 \sim 4$. Denote $\hat{d}_{j}$ as the estimations of $d_{j}^{*}$, and $\tilde{d}_{j}=d_{j}^{*}-\hat{d}_{j}, j=1,2$. When system (1-2) is operating at this point, the relation $P_{2}\left(\delta^{*}\right)+P_{3}\left(\delta^{*}\right) E_{f d}^{*}=P_{m} / T_{d 0}^{\prime}$ hold, $E_{f d}^{*}$ is constant and fixes the equilibrium value.

Step 1. Define $z_{1}=\delta-\delta^{*}$. Choose Lyapunov function $V_{1}=\left(c_{1} / 2\right) z_{1}^{2}$, the time derivative of $V_{1}$ along system trajectories is $\dot{V}_{1}=-p_{1} c_{1} z_{1}^{2}+c_{1} z_{1} z_{2}$, in which $c_{1}$ and $p_{1}$ are positive constants, $\alpha_{1}\left(z_{1}\right)=-p_{1} z_{1}$ is the virtual control of $\omega$ and $z_{2}=\omega-\alpha_{1}\left(z_{1}\right)$.

Step 2. Augment Lyapunov function as $V_{2}=V_{1}+\left(c_{2} / 2\right) z_{2}^{2}$, then $\dot{V}_{2}=-\sum_{i=1}^{2} p_{i} c_{i} z_{i}^{2}-c_{2}\left(\omega_{0} / H\right) z_{2} z_{3}-c_{2} z_{2} \hat{d}_{1} \tanh \left(z_{2} / \varepsilon_{1}\right)+$ $+c_{2} z_{2} d_{1}(t)$, the virtual control of $P_{e}$ is chosen as $\alpha_{2}\left(\overline{\mathbf{z}}_{2}\right)=$ $=P_{m}+\left(H / \omega_{0}\right)\left[k_{1} z_{1}+k_{2} z_{2}+\hat{d}_{1} \tanh \left(z_{2} / \varepsilon_{1}\right)\right]$, where $c_{2}, p_{2}$ and $\varepsilon_{1}$ are positive constants, $k_{1}=c_{1} / c_{2}+p_{1} D / H-p_{1}^{2}, k_{2}=p_{1}+$ $+p_{2}-D / H, z_{3}=P_{e}-\alpha_{2}\left(\overline{\mathbf{z}}_{2}\right)$.

Step 3. Consider Lyapunov function $V_{3}=V_{2}+\left(c_{3} / 2\right) z_{3}^{2}+$ $+(1 / 2) \sum_{j=1}^{2} \tilde{d}_{j}^{2}$. Differentiating $V_{3}$, one gets

$$
\begin{aligned}
\dot{V}_{3}= & -\sum_{i=1}^{3} p_{i} c_{i} z_{i}^{2}+c_{3} P_{3}(\delta) z_{3} z_{4} \\
& +c_{2} z_{2} d_{1}(t)-c_{2} z_{2} d_{1}^{*} \tanh \left(z_{2} / \varepsilon_{1}\right) \\
& +c_{3} z_{3} d_{2}(t)-c_{3} z_{3} d_{2}^{*} \tanh \left(z_{3} / \varepsilon_{2}\right) \\
& -\left(c_{3} H / \omega_{0}\right) f_{3}\left(z_{2}, z_{3}, \hat{d}_{1}\right) d_{1}(t) \\
& -\left(c_{3} H / \omega_{0}\right) f_{3}\left(z_{2}, z_{3}, \hat{d}_{1}\right) d_{1}^{*} \tanh \left(f_{3}\left(z_{2}, z_{3}, \hat{d}_{1}\right) / \varepsilon_{1}\right)
\end{aligned}
$$

the expecting action of $\operatorname{sat}\left(E_{f d}\right)$ is chosen as

$$
\begin{aligned}
\dot{\hat{d}}_{1}= & c_{2} z_{2} \tanh \left(z_{2} / \varepsilon_{1}\right) \\
& +c_{3}\left(H / \omega_{0}\right) f_{3}\left(z_{2}, z_{3}, \hat{d}_{1}\right) \tanh \left(f_{3}\left(z_{2}, z_{3}, \hat{d}_{1}\right) / \varepsilon_{1}\right) \\
\dot{\hat{d_{2}}=}= & c_{3} z_{3} \tanh \left(z_{3} / \varepsilon_{2}\right) \\
\alpha_{3}\left(\overline{\mathbf{z}}_{3}\right)= & \left\{-P_{1}(\delta, \omega) P_{e}-P_{2}(\delta)-k_{3} z_{1}\right. \\
& +\left[k_{4}+c_{2} \omega_{0} /\left(c_{3} H\right)\right] z_{2}-\left(k_{2}+p_{3}\right) z_{3} \\
& -\hat{d}_{2} \tanh \left(z_{3} / \varepsilon_{2}\right)-\left(H / \omega_{0}\right)\left[k_{2} \hat{d}_{1} \tanh \left(z_{2} / \varepsilon_{1}\right)\right. \\
& \left.-\tanh \left(z_{2} / \varepsilon_{1}\right) \dot{\hat{d}}_{1}-f_{1}\left(z_{2}, \hat{d}_{1}\right) f_{2}\left(\overline{\mathbf{z}}_{3}\right)\right] \\
& -\left(H / \omega_{0}\right)\left[k_{2}+f_{1}\left(z_{2}, \hat{d}_{1}\right)\right] * \\
& \left.* \hat{d}_{1}(t) \tanh \left(f_{3}\left(z_{2}, z_{3}, \hat{d}_{1}\right) / \varepsilon_{1}\right)\right\} / P_{3}(\delta)
\end{aligned}
$$

where $c_{3}, p_{3}$ and $\varepsilon_{2}$ are positive constants, $z_{4}=\operatorname{sat}\left(E_{f d}\right)-\alpha_{3}\left(\overline{\mathbf{z}}_{3}\right)$, $k_{3}=\left(H / \omega_{0}\right)\left(k_{1} p_{1}+k_{2} c_{1} / c_{2}\right), k_{4}=\left(H / \omega_{0}\right)\left(k_{1}-k_{2} p_{2}\right), f_{1}\left(z_{2}\right.$, $\left.\hat{d}_{1}\right)=\left(\hat{d}_{1} / \varepsilon_{1}\right) \operatorname{sech}^{2}\left(z_{2} / \varepsilon_{1}\right), f_{2}\left(\overline{\mathbf{z}}_{3}\right)=-\left(c_{1} / c_{2}\right) z_{1}-p_{2} z_{2}-\left(\omega_{0} / H\right)$ $z_{3}-\hat{d}_{1} \tanh \left(z_{2} / \varepsilon_{1}\right), f_{3}\left(z_{2}, z_{3}, \hat{d}_{1}\right)=\left[k_{2}+f_{1}\left(z_{2}, \hat{d}_{1}\right)\right] z_{3}$.

Step 4. The real excitation controller is designed as

$$
E_{f d}=E_{f d 1}=\alpha_{3}\left(\overline{\mathbf{z}}_{3}\right)
$$

Lemma 4. Consider two sets: $C_{1}\left(\overline{\mathbf{z}}_{\mathbf{3}}, \lambda\right)=\left\{\overline{\mathbf{z}}_{\mathbf{3}}\left|U_{B}<\right| \alpha_{3}\left(\overline{\mathbf{z}}_{\mathbf{3}}\right) \mid\right.$ $\left.\leq U_{B}+\lambda, \operatorname{sign}\left(z_{3} \alpha_{3}\left(\overline{\mathbf{z}}_{\mathbf{3}}\right)\right)=-1\right\}$ and $C_{2}\left(\overline{\mathbf{z}}_{\mathbf{3}}\right)=\left\{\overline{\mathbf{z}}_{\mathbf{3}} \mid P\left(\overline{\mathbf{z}}_{\mathbf{3}}\right)<p_{3}\right\}$, in which $\lambda$ named "saturation coefficient" is a given positive constant, $P\left(\overline{\mathbf{z}}_{\mathbf{3}}\right)=P_{3}(\delta)\left(\left|\alpha_{3}\left(\overline{\mathbf{z}}_{\mathbf{3}}\right)\right|-U_{B}\right) /\left|z_{3}\right|$. The set $C\left(\overline{\mathbf{z}}_{\mathbf{3}}, \lambda\right)=\left\{\overline{\mathbf{z}}_{\mathbf{3}}|| \alpha_{3}\left(\overline{\mathbf{z}}_{\mathbf{3}}\right) \mid \leq U_{B}+\lambda\right\}$ is a region of attraction if $C_{1}\left(\overline{\mathbf{z}}_{3}, \lambda\right) \subseteq C_{2}\left(\overline{\mathbf{z}}_{\mathbf{3}}\right)$.

Theorem 1. For SMIB power system which is expressed by the nonlinear dynamic equation (1-2) containing the influences of input saturation and time-varying uncertainties indicating the model errors, the excitation controller (5-6) ensures convergence of $\overline{\mathbf{z}}_{\mathbf{3}}$ to the origin under " $\lambda$ level saturation" if $\overline{\mathbf{z}}_{\mathbf{3}}(0) \in C\left(\overline{\mathbf{z}}_{\mathbf{3}}, \lambda\right)$.

Proof. Based on the designed controller (5-6), the following three cases are given to prove Lemma 4 and Theorem 1 .

Case 1. No saturation occurs, i.e. $\left|\alpha_{3}\left(\overline{\mathbf{z}}_{3}\right)\right| \leq U_{B}$. Invoking Lemma 3 directly for the equation (4), the following results can be obtained

$$
\dot{V}_{3} \leq-\sum_{i=1}^{3} p_{i} c_{i} z_{i}^{2}+\sigma
$$

where $\sigma=0.2785 \varepsilon_{1} d_{1}^{*}\left(c_{2}+c_{3} H / \omega_{0}\right)+0.2785 \varepsilon_{2} c_{3} d_{1}^{*}$.

Case 2. Saturation occurs, i.e. $U_{B}<\left|\alpha_{3}\left(\overline{\mathbf{z}}_{\mathbf{3}}\right)\right| \leq U_{B}+\lambda$, and in the meanwhile $\operatorname{sign}\left(z_{3} \alpha_{3}\left(\overline{\mathbf{z}}_{3}\right)\right) \geq 0$. Thus,

$$
\begin{aligned}
\dot{V}_{3} \leq & -\sum_{i=1}^{3} p_{i} c_{i} z_{i}^{2} \\
& +c_{3} P_{3}(\delta)\left|z_{3}\right| \operatorname{sign}\left(z_{3} \alpha_{3}\left(\overline{\mathbf{z}}_{3}\right)\right)\left(U_{B}-\left|\alpha_{3}\left(\overline{\mathbf{z}}_{3}\right)\right|\right)+\sigma \\
\leq & -\sum_{i=1}^{3} p_{i} c_{i} z_{i}^{2}+\sigma .
\end{aligned}
$$

Case 3. Saturation occurs, i.e. $U_{B}<\left|\alpha_{3}\left(\overline{\mathbf{z}}_{3}\right)\right| \leq U_{B}+\lambda$, and also the inequality $\operatorname{sign}\left(z_{3} \alpha_{3}\left(\overline{\mathbf{z}}_{\mathbf{3}}\right)\right)=-1$ holds, i.e. $\overline{\mathbf{z}}_{\mathbf{3}} \in C_{1}\left(\overline{\mathbf{z}}_{\mathbf{3}}, \lambda\right)$. In this case, one can get the following inequality

$$
\begin{aligned}
\dot{V}_{3} \leq & -\sum_{i=1}^{2} p_{i} c_{i} z_{i}^{2} \\
& -c_{3} P_{3}(\delta)\left|z_{3}\right|\left(p_{3}\left|z_{3}\right| / P_{3}(\delta)+U_{B}-\left|\alpha_{3}\left(\overline{\mathbf{z}}_{3}\right)\right|\right)+\sigma
\end{aligned}
$$


and furthermore, if the condition $C_{1}\left(\overline{\mathbf{z}}_{3}, \lambda\right) \subseteq C_{2}\left(\overline{\mathbf{z}}_{3}\right)$ holds, then there exists a constant $p>0$ such that

$$
\dot{V}_{3} \leq-\sum_{i=1}^{2} p_{i} c_{i} z_{i}^{2}-p c_{3} P_{3}(\delta)\left|z_{3}\right|+\sigma
$$

The results of the above three cases conclude the proof of Lemma 4 and Theorem 1.

Step 5. Note that, for given parameters $\lambda, p_{i}, c_{i}, \varepsilon_{j}, i=1,2,3$, $j=1,2$, how to estimate the region of attraction $C\left(\overline{\mathbf{z}}_{\mathbf{3}}, \lambda\right)$ is the key point. However, our goal in this paper is not to focus on this estimation, but instead to redesign the excitation control law (5-6) to stabilize the nonlinear system with input saturation. Then the proposed SAEC control strategy is presented as follows

$$
\begin{aligned}
L & = \begin{cases}\operatorname{sign}\left(P\left(\overline{\mathbf{z}}_{\mathbf{3}}\right)-\chi\right), & \overline{\mathbf{z}}_{\mathbf{3}} \in C_{1}\left(\overline{\mathbf{z}}_{\mathbf{3}}, \lambda\right), \\
-1, & \text { otherwise },\end{cases} \\
s & =\left\{\begin{array}{ll}
1, & \left\|\overline{\mathbf{z}}_{\mathbf{3}}\right\| \leq \tau \\
2, & \text { otherwise, }
\end{array} \text { or } L=-1,\right. \\
\rho \dot{\chi} & = \begin{cases}P\left(\overline{\mathbf{z}}_{\mathbf{3}}\right)-\chi+l_{\Delta}, & L \neq-1, \\
0, & \text { otherwise },\end{cases} \\
E_{f d 2} & =\alpha_{3}\left(\overline{\mathbf{z}}_{\mathbf{3}}, \chi\right)=\left.\alpha_{3}\left(\overline{\mathbf{z}}_{\mathbf{3}}\right)\right|_{p_{3}=\chi}, \\
E_{f d} & =E_{f d s},
\end{aligned}
$$

where the positive constants $\rho, \tau$ and $l_{\Delta}$ are parameters to be tuned appropriately by the engineer, $\chi(0)=p_{3}$.

Theorem 2. There exist small positive parameters $\rho, \tau$ and $l_{\Delta}$ such that, in the presence of input saturation and time-varying uncertainties, the controller (5), (11) achieves global stability of SMIB power systems (1-2) and the transient performances are improved by using the switching control strategy.

Proof. In general, with appropriate choice of $\tau$, the inequalities $\left|E_{f d 1}\right| \leq U_{B}$ and $\left\|\overline{\mathbf{z}}_{\mathbf{3}}\right\| \leq \tau$ are equivalent.

In Case 1 and Case 2: one can get $E_{f d}=E_{f d 1}$.

In Case 3, if $\overline{\mathbf{z}}_{\mathbf{3}} \in C_{1}\left(\overline{\mathbf{z}}_{\mathbf{3}}, \lambda\right)$ and $\left.\overline{\mathbf{z}}_{\mathbf{3}} \notin C_{2}\left(\overline{\mathbf{z}}_{\mathbf{3}}\right)\right|_{p_{3}=\chi}$ i.e. $P\left(\overline{\mathbf{z}}_{\mathbf{3}}\right) \geq \chi$ : the logic judgment of the auxiliary system in (11) can activate the dynamic system $\rho \dot{\chi}=P\left(\overline{\mathbf{z}}_{3}\right)-\chi+l_{\Delta}$. As such, since $\rho$ is small, the variable $\chi$ can reach a small neighborhood of the manifold $\chi=P\left(\overline{\mathbf{z}}_{\mathbf{3}}\right)+l_{\Delta}$, then $P\left(\overline{\mathbf{z}}_{\mathbf{3}}\right)<\chi$ and the condition $\left.\overline{\mathbf{z}}_{\mathbf{3}} \in C_{2}\left(\overline{\mathbf{z}}_{\mathbf{3}}\right)\right|_{p_{3}=\chi}$ holds.

Consequently, on the basis of Theorem 1 and Lemma 4, the convergence of $\overline{\mathbf{z}}_{\mathbf{3}}$ to the origin can be ensured.

Therefore, the main advantages of sub-controllers $E_{f d 1}$ and $E_{f d 2}$ are realized in Cases 1, 2 and Case 3 respectively. Thus, the switching strategy is proposed to make logical choice between them based on the power systems operating conditions. As such, the transient performances are improved.
3.3. Discussion. In the above subsection, the SAEC control scheme is proposed to ensure stability of power systems even if there is control input saturation and time-varying uncertainties. But since the auxiliary dynamic system and switching strategy are needed in SAEC, the cost of control system is often more than that of general linear control system. Thus, an alternative control scheme is shown in this subsection to facilitate engineers to trade off between cost and performance: a sufficient condition for allowing $\gamma$-saturation of a kind of linear excitation control input is presented, and a method for the estimation of the region of attraction is given.

Next, the system (1-2) is equivalently transformed into an error system. Let $e_{i}$ be the state variables of this error system and $\overline{\mathbf{e}}_{\mathbf{i}}=\left[e_{1}, \ldots, e_{i}\right]^{\mathrm{T}}, i=1 \sim 3$.

Step I. Define $e_{1}=\delta-\delta^{*}$. The time derivative of $e_{1}$ along system trajectories is $\dot{e}_{1}=-q_{1} e_{1}+e_{2}$, in which $q_{1}$ is positive constant, $\beta_{1}\left(e_{1}\right)=-q_{1} e_{1}$ is the virtual control of $\omega$ and $e_{2}=\omega-\beta_{1}\left(e_{1}\right)$.

Step II. Then $\dot{e}_{2}=-\left(q_{2}+r_{2}\right) e_{2}-\left(\omega_{0} / H\right) e_{3}+d_{1}(t)$, the virtual control of $P_{e}$ is chosen as $\beta_{2}\left(\overline{\mathbf{e}}_{\mathbf{2}}\right)=P_{m}+\left(H / \omega_{0}\right)\left(r_{1} e_{1}+q_{2} e_{2}\right)$, where $q_{2}$ is positive constant, $r_{1}=q_{1} D / H-q_{1}^{2}, r_{2}=D / H-q_{1}$, $e_{3}=P_{e}-\beta_{2}\left(\overline{\mathbf{e}}_{2}\right)$.

Step III. Differentiating $e_{3}$, one gets $\dot{e}_{3}=-\left(q_{3}+1 / T_{d 0}^{\prime}-q_{2}\right)$ $e_{3}+u-\mathbf{r} \overline{\mathbf{e}}_{3}+d_{z 2}$, where $q_{3}$ is positive constant, $r_{3}=r_{1} H\left(q_{1}-\right.$ $\left.-1 / T_{d 0}^{\prime}\right) / \omega_{0}, r_{4}=\left(H / \omega_{0}\right)\left(r_{1}-q_{2}^{2}-q_{2} r_{2}+q_{2} / T_{d 0}^{\prime}\right), \mathbf{r}=\left[-r_{3} r_{4}-q_{3}\right]$, $d_{z 1}=d_{1}(t), d_{z 2}=P_{2}(\delta)-P_{2}\left(\delta^{*}\right)+\omega P_{e} \cot \delta+d_{2}(t)-\left(q_{2}\right.$ $\left.H / \omega_{0}\right) d_{1}(t), u=P_{3}(\delta) s a t\left(E_{f d}\right)-P_{3}\left(\delta^{*}\right) E_{f d}^{*}$ represents the control variable of error system to be designed later.

Step IV. The saturation on $E_{f d}$ can be equivalently transformed into the constant constraint $u_{c}$ on $u$, namely, $|u| \leq u_{c} \leq$ $\leq U_{B} P_{3}\left(\delta_{m}\right)-P_{3}\left(\delta^{*}\right) E_{f d}^{*}$. Generally speaking, for $\gamma \in(0,1]$, if $\gamma$-saturation happens to $E_{f d}$, there must exist a constant $\varsigma \in(0,1]$ such that $|u| \leq u_{c} / \varsigma \leq U_{B} P_{3}\left(\delta_{m}\right) / \gamma-P_{3}\left(\delta^{*}\right) E_{f d}^{*}$, correspondingly, $\varsigma$-saturation happens to $u$. Based on the above analysis, let $u$ be specified as $u=\mathbf{r} \overline{\mathbf{e}}_{3}$, and the real excitation control input is designed as $E_{f d}=\left[\mathbf{r} \overline{\mathbf{e}}_{\mathbf{3}}+P_{3}\left(\delta^{*}\right) E_{f d}^{*}\right] / P_{3}(\delta)$, then, one can obtain the following error system:

$$
\text { no-saturation: } \quad \dot{\overline{\mathbf{e}}}_{\mathbf{3}}=\mathbf{A} \overline{\mathbf{e}}_{\mathbf{3}}+\mathbf{D} \mathbf{d}_{\mathbf{z}} \text {, }
$$

$$
\text { ऽ-saturation: } \quad \dot{\overline{\mathbf{e}}}_{\mathbf{3}}=\mathbf{A} \overline{\mathbf{e}}_{\mathbf{3}}+(\varsigma-1) \mathbf{b r} \overline{\mathbf{e}}_{\mathbf{3}}+\mathbf{D d}_{\mathbf{z}} \text {, }
$$

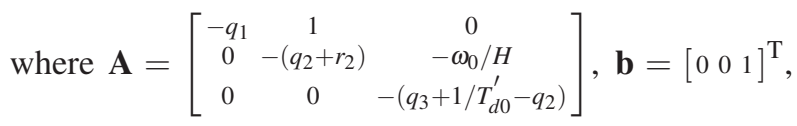

$$
\begin{aligned}
& \mathbf{D}=\left[\begin{array}{lll}
0 & 1 & 0 \\
0 & 0 & 1
\end{array}\right]^{\mathrm{T}}, \mathbf{d}_{\mathbf{z}}=\left[\begin{array}{ll}
d_{z 1} & d_{z 2}
\end{array}\right]^{\mathrm{T}} \text {. }
\end{aligned}
$$

Step V. Choose Lyapunov function as $V_{e}=(1 / 2) \overline{\mathbf{e}}_{\mathbf{3}}^{\mathbf{T}} \mathbf{Q} \overline{\mathbf{e}}_{\mathbf{3}}$, where $0<\mathbf{Q}=\mathbf{Q}^{\mathbf{T}} \in \mathbb{R}^{3 \times 3}$ are to be chosen later. Thus the time derivative of $V_{e}$ along the system trajectories is 


$$
\begin{aligned}
\dot{V}_{e}= & \overline{\mathbf{e}}_{\mathbf{3}}^{\mathrm{T}} \mathbf{A}_{\mathbf{k}}^{\mathrm{T}} \mathbf{Q} \overline{\mathbf{e}}_{\mathbf{3}}+\overline{\mathbf{e}}_{\mathbf{3}}^{\mathrm{T}} \mathbf{Q D D d} \mathbf{z} \\
= & \overline{\mathbf{e}}_{\mathbf{3}}^{\mathrm{T}}\left[\mathbf{A}_{\mathbf{k}}^{\mathrm{T}} \mathbf{Q}+\left(1 /\left(2 \varepsilon^{2}\right)\right) \mathbf{Q D D D} \mathbf{D}^{\mathrm{T}} \mathbf{Q}\right] \overline{\mathbf{e}}_{\mathbf{3}} \\
& -(1 / 2)\left\|\boldsymbol{\varepsilon} \mathbf{d}_{\mathbf{z}}-(1 / \varepsilon) \mathbf{D}^{\mathrm{T}} \mathbf{Q}_{\mathbf{\mathbf { e }}} \overline{\mathbf{H}}^{2}+(1 / 2) \varepsilon^{2}\right\| \mathbf{d}_{\mathbf{z}} \|^{2},
\end{aligned}
$$

where $\mathbf{A}_{\mathbf{k}}=\mathbf{A}$ when there is no saturation, and $\mathbf{A}_{\mathbf{k}}=\mathbf{A}_{\mathbf{k} \varsigma}=\mathbf{A}+$ $+(\varsigma-1) \mathbf{b r}$ when there is $\varsigma$-saturation, $\varepsilon$ is disturbance attenuation constant.

Theorem 3. For the error dynamic system (12-13) and given $\varsigma \in(0,1], \rho>0,0<\varepsilon<1$, the control input $u=\mathbf{r e}_{\mathbf{3}}$ ensures convergence of $\overline{\mathbf{e}}_{\mathbf{3}}$ to a ball of radius $\varepsilon$ under $\varsigma$-saturation if there exist $\mathbf{Q}$ satisfying

$$
\begin{array}{r}
\mathbf{A}^{\mathrm{T}} \mathbf{Q}+\left(1 /\left(2 \varepsilon^{2}\right)\right) \mathbf{Q D D} \mathbf{D}^{\mathrm{T}} \mathbf{Q}<0, \\
\mathbf{A}_{\mathbf{k} \varsigma}{ }^{\mathrm{T}} \mathbf{Q}+\left(1 /\left(2 \varepsilon^{2}\right)\right) \mathbf{Q D D} \mathbf{D}^{\mathrm{T}} \mathbf{Q}<0, \\
{\left[\begin{array}{cc}
\mathbf{Q} & \varsigma_{\mathbf{T}}^{\mathrm{T}} \\
\varsigma \mathbf{r}\left(u_{c}^{2} / \rho\right)
\end{array}\right] \geq 0,}
\end{array}
$$

and then the region of attraction can be estimated by using the following inequality

$$
\left[\begin{array}{cc}
\rho & \overline{\mathbf{e}}_{3}(0)^{\mathrm{T}} \\
\overline{\mathbf{e}}_{\mathbf{3}}(0) & \mathbf{Q}^{-1}
\end{array}\right] \geq 0 .
$$

Proof. The objective of control design is to guarantee the decay of Lyapunov function $V_{e}$ whether $\varsigma$-saturation happens or not. Thus, from equation (14) and Lemma 2 , one can conclude this proof.

Remark 1. The main differences between SAEC and the linear excitation control in this subsection are: (i) the former is of initiative control action from the perspective of "anti-saturation", but the latter is of passive system analysis from the viewpoint of "estimation of region of attraction" allowing $\varsigma$-saturation. (ii)
Lyapunov function $V_{3}$ is obtained by employing constructive design, and the control gain $p_{1}, p_{2}, p_{3}$ of SAEC can be specified according to practical operating condition. However, only the structure of the Lyapunov function $V_{e}$ is known, the matrix $Q$ can be got by solving the LMI (15). Also, in order to decrease control gain, the inherent damping coefficients of power systems are reserved such as $-D / H,-1 / T_{d 0}^{\prime}$. (iii) For the timevarying uncertainties, adaptive laws are proposed in SAEC, and a disturbance attenuation condition is give in the latter scheme.

From the above discussion, the advantages of the SAEC are: (i) global convergence can be ensured when there are control input saturation and time-varying uncertainties. (ii) better performance is obtained than that of linear controller since it has adaptive mechanism and auxiliary decision system. The main characteristics of the linear controller are: (i) lower costs than that of SAEC, only off-line analysis is needed to obtain the region of attraction considering $\varsigma$-saturation and time-varying uncertainties. (ii) it is suitable for power systems under small signal disturbances.

\section{Simulation results}

In this section, the developed control strategy for a SMIB power system is shown to be effective by a comparative simulation study of the SAEC and the excitation controller which is designed by using the approach proposed in [17]. The transient responses of power angle, relative speed, active power and control input are shown in Figs 2-5 with the same initial condition $\delta(0)=1.309\left(75^{\circ}\right), \omega(0)=5, P_{e}(0)=0.7$ and also with the same limit on the control input $E_{f d}$. The values of the parameters and operating point used in the simulation are respectively listed as follows: 1) Values of system parameters: $\omega_{0}=314.159, D=5$, $H=7.1, V_{s}=1, x_{d}=1.79, x_{d}^{\prime}=0.17, x_{T}=0.02, x_{L}=0.8$, $x_{d s}^{\prime}=x_{d}^{\prime}+x_{T}+x_{L}=0.99, T_{d 0}^{\prime}=7.65, T_{d 0}=5, U_{B}=6$; 2) Operating point: $\left.\left(\delta, \omega, P_{e}\right)=(0.3475,0,0.81) ; 3\right)$ Values of designed parameters: $c_{1}=c_{2}=c_{3}=1, p_{1}=p_{2}=p_{3}=0.5$,

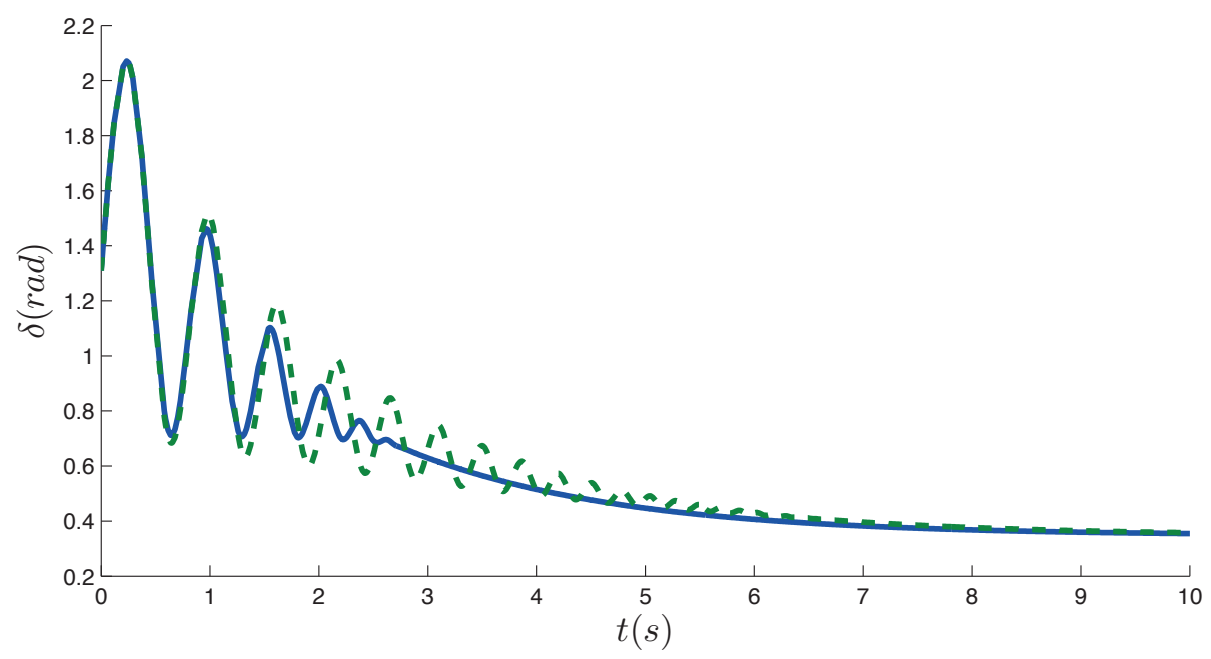

Fig. 2. Transient response curves of the power angle 


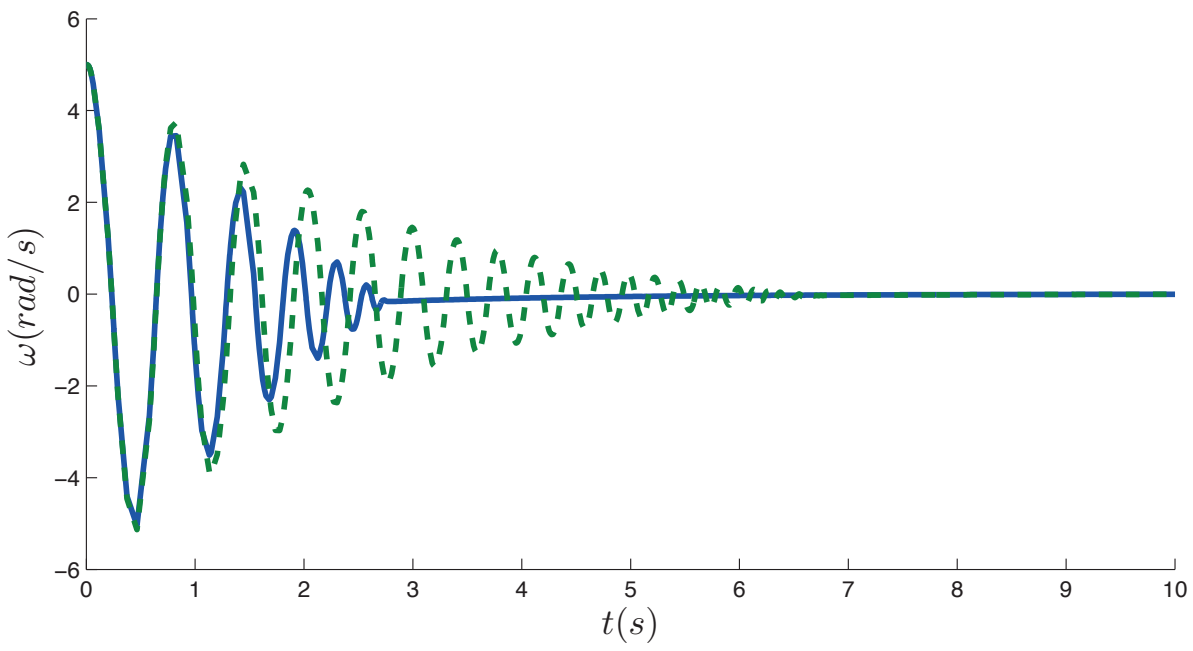

Fig. 3. Transient response curves of the relative speed

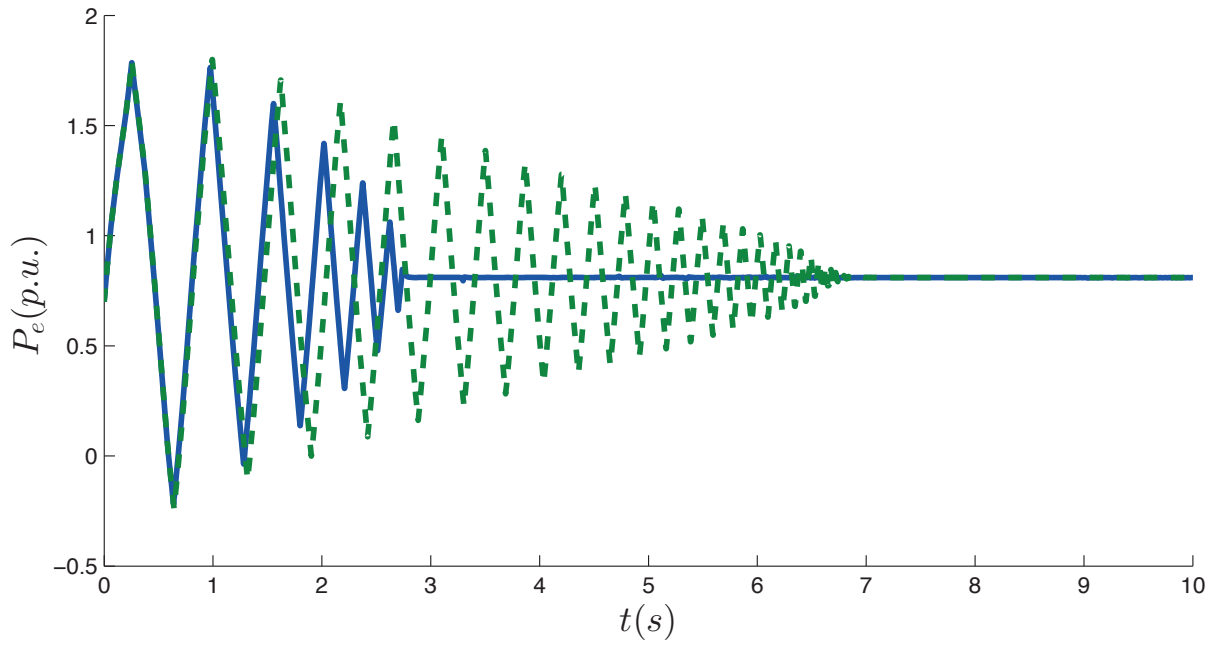

Fig. 4. Transient response curves of the active power

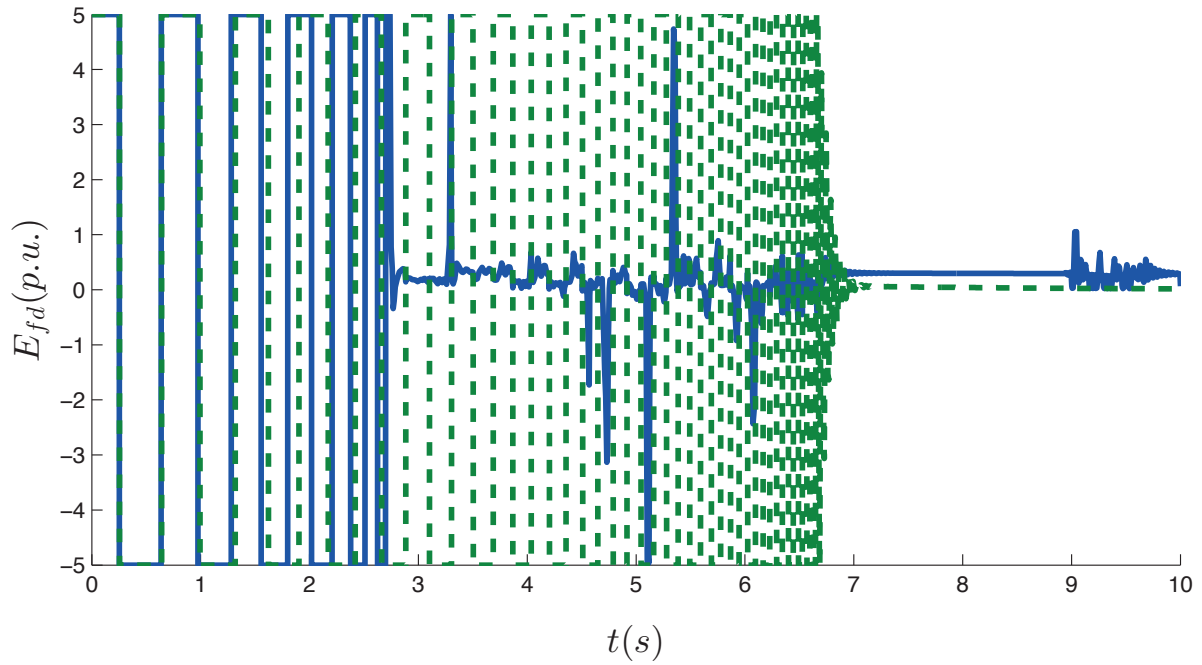

Fig. 2. Transient response curves of the power angle 
$\varepsilon_{1}=\varepsilon_{2}=0.05, \rho=0.5, \tau=2, l_{\Delta}=0.1$; 4) Time-varying uncertainties: $d_{1}(t)=2 \sin (\omega t), d_{2}(t)=0.03 \sin (0.01 \omega t)$.

In Figs 2-5, the solid lines and the dash lines represent the results with the proposed SAEC and the controller in [17] respectively. From these figures, the SAEC globally stabilizes the system (1-2) and provides better performance than the controller given in [17], which does not consider the influence of input saturation. The simulation results verify the proposed theoretical results i.e. Theorem 1 and Theorem 2, and the superiority of the proposed control method over existing methodologies that ignore input saturation within the design is demonstrated.

\section{Conclusions}

A new control synthesis framework was developed to design a kind of nonlinear robust generator excitation controller for power systems. Compared with the conventional control schemes, by employing adaptive auxiliary decision mechanism, global convergence and better transient performances have been achieved theoretically and simulatively even with control input saturation and time-varying uncertainties.

The practicability of the proposed control law is discussed as follows: firstly, as a model-based approach, it is important to verify the accuracy of the model used in this paper. Fortunately, as stated in Introduction, the SMIB model has been validated to be well-suited for power system analysis and control. Moreover, time-varying uncertainties have been considered, which has increased the exactness of the classical SMIB model. Secondly, only local measurable information is needed for the designed control law in which the parameters can be tuned flexibly in terms of practical operating conditions and engineering requirements.

Acknowledgements. This work was supported by the National Natural Science Foundation of China under Grants 61403194, the Natural Science Foundation of Jiangsu Province under Grant BK20140836, the Foundation of Jiangsu Key Laboratory "Internet of Things and Control Technologies" and NUAA Research Funding for Introduction of Talent under Grant 1003-56YAH14007.

\section{REFERENCES}

[1] P. M. Anderson and A. A. Fouad, Power System Control and Stability, Second Edition, Wiley-IEEE Press, 2002

[2] G. M. Dimirovski, Y.W. Jing,W. L. Li, and X. P. Liu, "Adaptive back-stepping design of tcsc robust nonlinear control for power systems," Intelligent Automation \& Soft Computing 12 (1), 75- 87 (2006).

[3] M. Galaz, R. Ortega, A. S. Bazanella, and A.M. Stankovic, "An energy-shaping approach to the design of excitation control of synchronous generators," Automatica 39 (1), 111-119 (2003).
[4] S. Mei, Y. Ni, G. Wang, and S. Wu, "A study of self-organized criticality of power system under cascading failures based on ac-opf with voltage stability margin," IEEE Transactions on Power Systems, 23 (4), 1719-1726 (2008).

[5] P. Pruski and S. Paszek. "Assessment of Polish Power System angular stability based on analysis of different disturbance waveforms," Bull. Pol. Ac.: Tech 63 (2), 435-441 (2015).

[6] A. Isidori, Nonlinear Control Systems, Springer Science\&Business Media, 1995.

[7] S. Mei, T. Shen, and K. Liu, Modern Robust Control Theory and Application, Beijing: Tsinghua, 2003.

[8] L. Y. Sun, S. Tong, and Y. Liu, "Adaptive backstepping sliding mode control of static var compensator," IEEE Transactions on Control Systems Technology 19 (5), 1178-1185 (2011).

[9] Y. Li, T. Li, and X. Jing, "Indirect adaptive fuzzy control for input and output constrained nonlinear systems using a barrier lyapunov function," International Journal of Adaptive Control and Signal Processing 28 (2), 184-199 (2014).

[10] M. Chen, G. Tao, and B. Jiang, "Dynamic surface control using neural networks for a alass of uncertain nonlinear systems with input saturation," IEEE Transactions on Neural Networks and Learning Systems 26 (9), 2086-2097 (2014).

[11] B. Song and J. K. Hedrick, "Simultaneous quadratic stabilization for a class of non-linear systems with input saturation using dynamic surface control," International Journal of Control 77 (1), 19-26 (2004).

[12] S. Boyd, L. El Ghaoui, E. Feron, and V. Balakrishnan, Linear Matrix Inequalities in System and Control Theory, Philadelphia, SIAM, 1994.

[13] W. Dib, A. E. Barabanov, R. Ortega, and F. Lamnabhi- Lagarrigue, "An explicit solution of the power balance equations of structure preserving power system models," IEEE Transactions on Power Systems 24 (2), 759-765 (2009).

[14] W. Dib, R. Ortega, A. Barabanov, and F. Lamnabhi-Lagarrigue, "A globally convergent controller for multi-machine power systems using structure-preserving models," IEEE Transactions on Automatic Control 54 (9), 2179-2185 (2009).

[15] M. A. Arjona, R. Escarela-Perez, G. Espinosa-Perez, and J. Alvarez-Ramirez, "Validity testing of third-order nonlinear models for synchronous generators," Electric Power Systems Research 79 (6), 953-958 (2009).

[16] P. V. Kokotovic and P.W. Sauer, "Integral manifold as a tool for reduced-order modeling of nonlinear systems: A synchronous machine case study," IEEE Transactions on Circuits and Systems 36 (3), 403-410 (1989).

[17] T. Shen, S. Mei, Q. Lu, W. Hu, and K. Tamura, "Adaptive nonlinear excitation control with 12 disturbance attenuation for power systems," Automatica 39 (1), 81-89 (2003).

[18] Y.Wan and J. Zhao, "Extended backstepping method for singlemachine infinite-bus power systems with smes," IEEE Transactions on Control Systems Technology 21 (3), 915-923 (2013).

[19] G. Gurrala and I. Sen, "Power system stabilizers design for interconnected power systems," IEEE Transactions on Power Systems 25 (2), 1042-1051 (2010).

[20] X. Jin, "Adaptive fault tolerant control for a class of input and state constrained mimo nonlinear systems," International Journal of Robust and Nonlinear Control 26 (2), 286-302 (2016). 